\title{
Quantitative trait loci for callus initiation and totipotency in maize (Zea mays L.)
}

\author{
M. D. Krakowsky $\cdot$ M. Lee $\cdot$ L. Garay • \\ W. Woodman-Clikeman $\cdot$ M. J. Long • \\ N. Sharopova $\cdot$ B. Frame $\cdot$ K. Wang
}

Received: 11 January 2006 / Accepted: 3 June 2006 / Published online: 8 August 2006

(C) Springer-Verlag 2006

\begin{abstract}
Induction of embryogenic callus in culture is an important step in plant transformation procedures, but response is genotype specific and the genetics of the trait are not well understood. Quantitative trait loci (QTL) were mapped in a set of 126 recombinant inbred lines (RILs) of inbred H99 (high Type I callus response) by inbred Mo17 (low Type I callus response) that were evaluated over two years for Type I callus response. QTL were observed in a total of eleven bins on eight chromosomes, including eight QTL with main effects and three epistatic interactions. Many of the QTL were mapped to the same or bordering chromosomal bins as candidate genes for abscisic acid metabolism, indicating a possible role for the hormone in the induction of embryogenic callus, as has previously been indicated in microspore embryo induction. Further examinations of allelic variability for known candidate genes located near the observed QTL could be useful for expanding the understanding of the genetic basis of induction embryogenic callus. The QTL observed herein could also be used in a marker assisted selection (MAS) program to improve the
\end{abstract}

Communicated by P. Langridge

M. D. Krakowsky · M. Lee $\cdot$ L. Garay

W. Woodman-Clikeman · M. J. Long · N. Sharopova •

B. Frame $\cdot$ K. Wang

Department of Agronomy, Iowa State University,

Ames, IA 50011, USA

M. D. Krakowsky $(\square)$

United States Department of Agriculture,

Agricultural Research Service, Tifton,

GA 31793, USA

e-mail:mkrakowsky@tifton.usda.gov response of agronomically useful inbreds, but only if the resources required for MAS are lower than those required for phenotypic selection.

\section{Introduction}

The potential of germplasm to form callus in culture and regenerate plants is a necessary prerequisite for plant transformation procedures, though the genetic processes underlying these traits are not well understood in maize. Green and Philips (1975) initially described the regeneration of maize plants from callus derived from immature embryos of maize inbred A188, a process that was facilitated by the placement of the plumule-radicle axis side of the embryo in contact with the media used for culture. Further studies distinguished two types of maize callus, identified as Type I and Type II (Green and Rhodes 1982; Lu et al. 1982). Type I callus is compact and more easily obtained from immature embryos, while Type II is friable and maintains the ability to regenerate plants over a longer period of time. The different callus types may originate from the same initial cells within the scutella of immature embryos, with the observed differences due to genotype, stage of immature embryo development, and culture media (Phillips et al. 1988).

The ability to produce regenerable callus from immature embryos appears to be genotype-dependent in both temperate and tropical maize (Tomes and Smith 1985; Hodges et al. 1986; Carvalho et al. 1997). Efficiencies of regenerable callus production are low for many agronomically useful, publicly available inbreds (e.g., B73, Mo17), which can create the need to use less desirable inbreds (e.g., A188, H99) for genetic transformation and 
plant regeneration (Hodges et al. 1986). This requires the use of several generations of backcrossing to produce agronomically desirable maize inbreds with the added trait of interest, a time frame that could be reduced through the use of regenerable elite germplasm.

The genetic basis of regenerable callus production has not been well examined in maize, with some early indications that a few simply inherited genes were of major importance (Hodges et al. 1985; Willman et al. 1989). Other reports suggest that additive, non-additive, and maternal effects significantly affect the induction of regenerable callus, although the importance of maternal effects is not consistent across reports (Beckert and Qing 1984; Duncan et al. 1985; Tomes 1985; Hodges et al. 1986). Only one report concerning the identification of genomic regions associated with induction of embryogenic callus from immature embryos has been published. Three of the five genomic regions of A188 that were conserved in backcross $(\mathrm{BC})$ lines of $(\mathrm{A} 188 \times \mathrm{B} 73)$ $\times$ B73 that were selected for high frequency initiation of embryogenic cultures were also significantly associated with percentage of immature embryos producing embryogenic callus in an $\mathrm{A} 188 \times \mathrm{Mo} 17 \mathrm{~F}_{2}$ population, on chromosomes 1, 4, and 9 (Armstrong et al. 1992). More research has focused on the genetics of anther culture response, with genomic regions associated with embryo-like structure (ELS) formation from microspores observed on all chromosomes (Cowen et al. 1992; Wan et al. 1992; Murgineux et al. 1994; Beaumont et al. 1995). Despite the differences in the explants there may be some minor similarities in the genetics of callus production from immature embryos and anthers. For example, maize inbred $\mathrm{H} 99$, which is highly efficient at producing embryogenic callus from immature embryos but is not responsive to anther culture, appears to have alleles that are positively associated with the induction of embryos during anther culture (Hodges et al. 1986; Beaumont et al. 1995).

Building on the limited base of knowledge concerning the genetics of regenerable callus production from immature embryos could be useful for not only improving tissue culture response of elite germplasm but also for expanding available information on the processes involved in the induction of regenerable callus. The objective of this research was to map quantitative trait loci (QTL) associated with induction of regenerable callus in recombinant inbred lines (RILs) of Mo17 × H99.

\section{Materials and methods}

Formation of the Mo17 $\times$ H99 RIL population has been described by Veldboom et al. (1994) and Austin and Lee (1996). Briefly, one $F_{1}$ plant was self-pollinated to produce $\mathrm{F}_{2}$ individuals that were advanced by the single-seed descent method to the $\mathrm{F}_{6}$ generation. Single-row plots, $3.8 \mathrm{~m}$ long and spaced $0.76 \mathrm{~m}$ apart, were planted for each RIL at the Agronomy and Agricultural Engineering Research Center (AAERC) near Ames, IA in a completely randomized design with one replication each in 1997 and 1998. All plants were selfpollinated by hand and approximately ten ears per RIL were harvested when the embryos were $0.75-1.0 \mathrm{~mm}$ long (about 6-10 days after pollination). Immature embryos were aseptically isolated from surface sterilized ears and cultured with the scutellum-side up on N6 medium as described in Armstrong et al. (1992).

The phenotype for each embryo was recorded as presence or absence of Type I callus after 4 weeks. The trait value for each entry was calculated as the percentage of embryos with Type I callus out of the total number of embryos isolated for the entry. Square-root transformations were performed on the data to reduce the effects of outlying values (see Figs. 1, 2) though both transformed and non-transformed data were analysed. RIL means were used for the individual year analyses, while least square means (LSM) for the combined analysis were calculated from the non-transformed and transformed values from both years using SAS Proc Mixed (SAS Institute 1999), considering entries as fixed effects. Genotype, genotype $\times$ environment, and error variances were estimated using a model that considered entries and the entry $\times$ environment interaction as random effects (Cardinal et al. 2003). Broad-sense heritabilities on an entry-mean basis and their exact confidence intervals were calculated according to established procedures (Knapp et al. 1985; Fehr 1987).

\section{Detection of QTL}

The genetic map for the population has been published (Austin and Lee, 1998). A subset of 78 RFLP loci and 33 SSR loci was used herein to reduce the number of tightly linked loci used in the QTL analysis. QTL were detected using PlabQTL with cofactor selection performed as described in Utz and Melchinger (1996) and Austin et al. (2000). Outlier or influential observations were tested based on statistics calculated by PlabQTL (Andrews-Pregibon statistic second factor, AP $2<0.5$; influential value of an observation, infl $>0.4$; Studentized residual, stdRes $>3.5$ ). The LOD threshold value of 2.5 was used to declare the presence of a QTL. This LOD threshold has been used in other studies of QTL in maize (Cardinal et al. 2003; Krakowsky et al. 2004) and has the advantage of minimizing the risk of a Type II error (i.e., missing a QTL). 


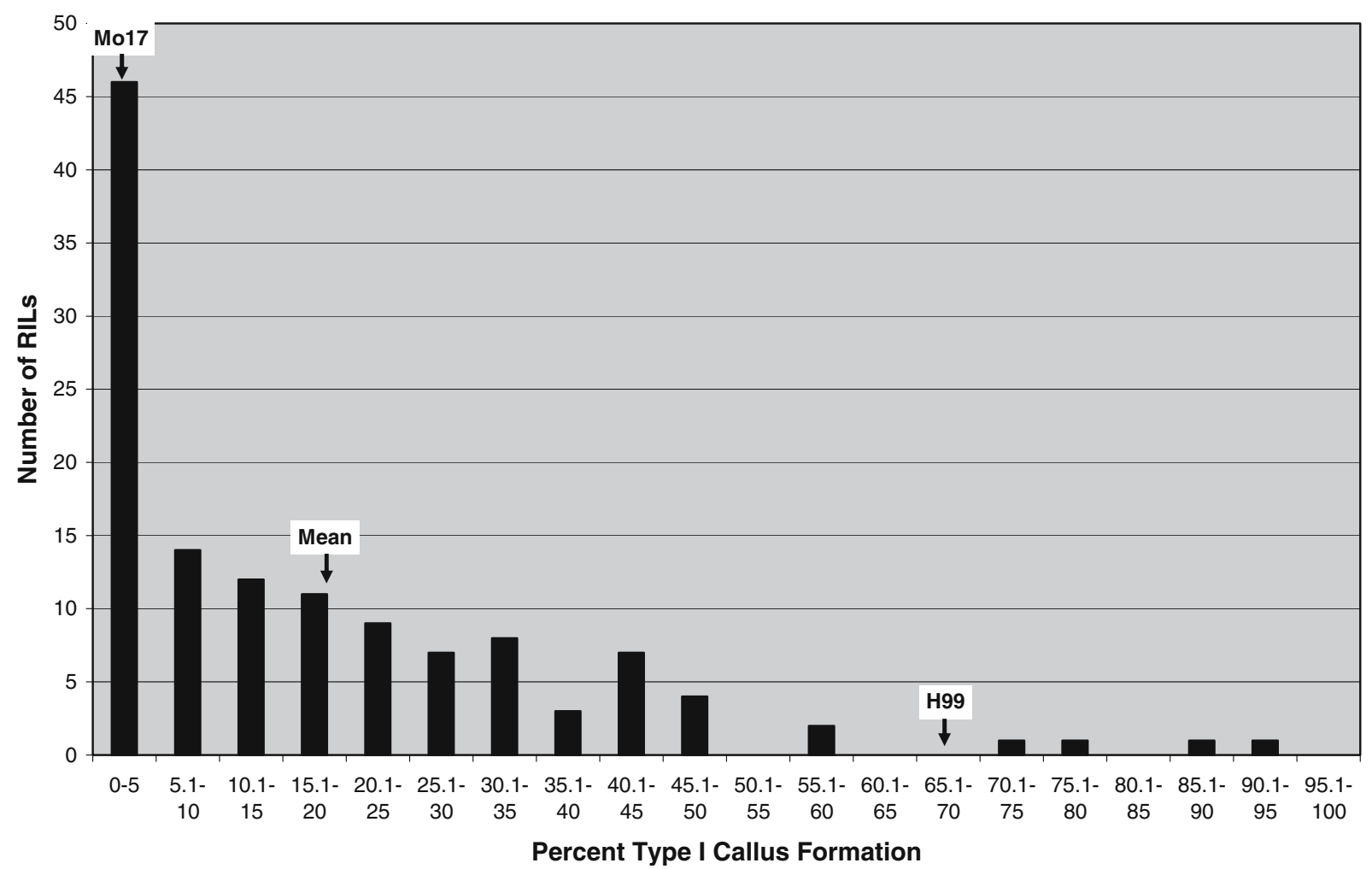

Fig. 1 Percent Type I callus formation, mean across trials (non-transformed data)

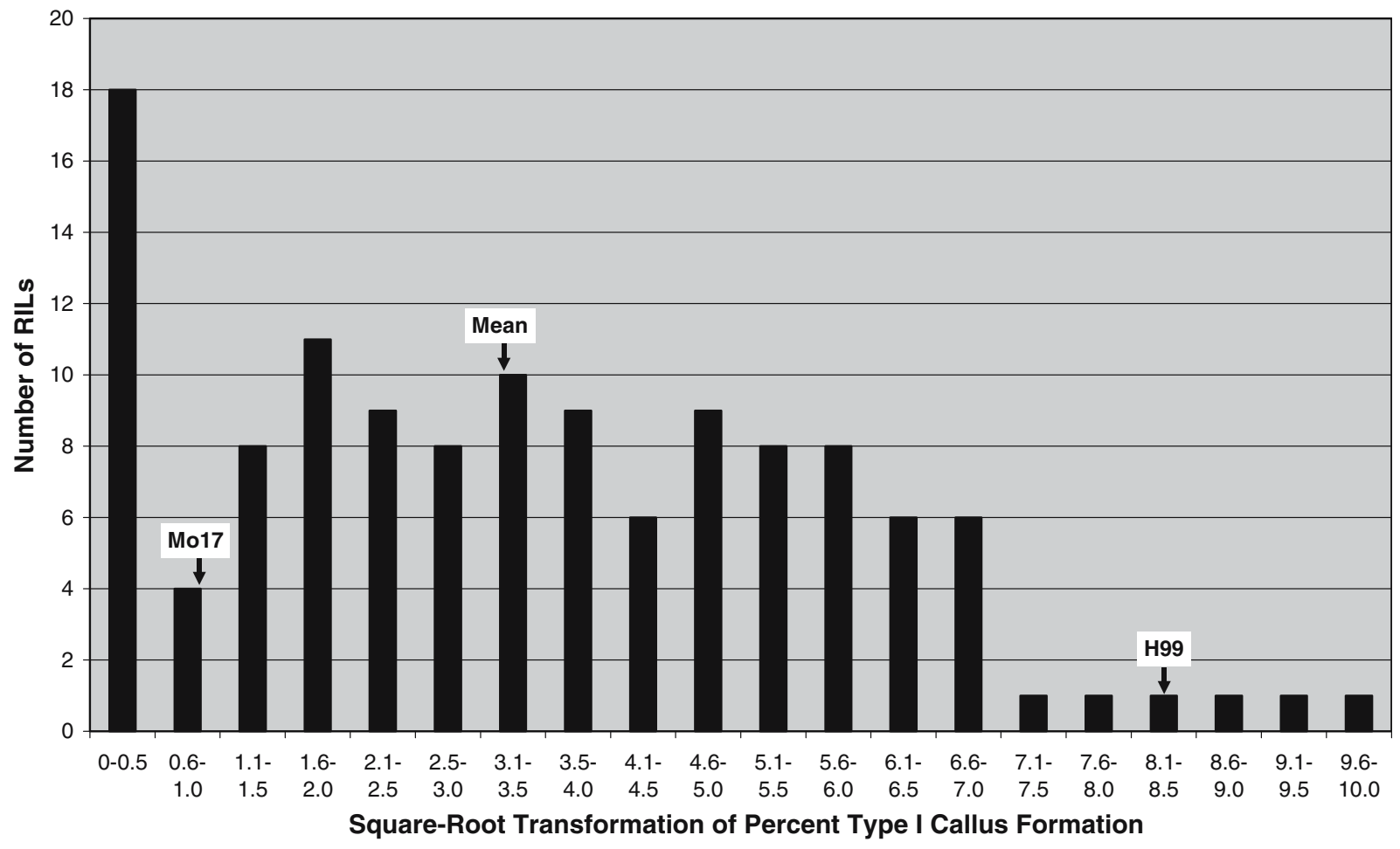

Fig. 2 Percent Type I callus formation, mean across trials (non-transformed data)

Digenic epistatic interactions between all pairs of loci were tested using Epistacy, which uses least-square statistics (Holland 1998). Interactions at $P<0.00026$ were considered significant. This threshold was based on an estimate of the number of independent linkage groups in maize with each chromosome arm representing one 
independent linkage group (Holland et al. 1997). Interaction terms were added to a model including all main effect QTL in PlabQTL, and those interactions that increased the AIC by at least 2.0 were deemed significant. Interactions between main effect QTL were also tested in PlabQTL by including additive $\times$ additive epistatic effects in the model. Effects that exceeded the Fto-Enter (and F-to-Drop) value obtained by using the Bonferroni bound at the significance level $(P)$ of 0.05 (experiment-wise error rate) were included in the final model.

The relative efficiency of marker assisted selection (MAS) over conventional phenotypic selection was calculated as described in Lande and Thompson (1990) and Papst et al. (2004), with the efficiency of selection based solely on MAS calculated as RE $=\sqrt{ } p / h^{2}$ and the efficiency of selection based on MAS and phenotypic data calculated as $\mathrm{RE}=\sqrt{ } p / h^{2}+(1-p)^{2} /\left(1-p h^{2}\right)$, where $h^{2}$ is the heritability of the trait and $p$ is the proportion of the additive genetic variance associated with the marker loci.

\section{Results}

Phenotypic analysis

The mean, median, and range of embryos evaluated per RIL were 78, 73, and 24-249, respectively, in 1997 and 71, 70, and 25-120, respectively, in 1998. The number of embryos evaluated per parental line was 404 and 374 in 1997 and 1998, respectively, for Mo17 and 319 and 240 in 1997 and 1998, respectively, for H99. Significant differences were observed between the inbred parents, with $71 \%$ of the H99 embryos and $1 \%$ of the Mo17 embryos forming Type I callus. In 1997 the mean response for the RILs was $21 \%$, with 36 entries exhibiting no response and 20 entries exhibiting greater than $50 \%$ response, while in 1998 the mean response was $14 \%$ with 52 entries exhibiting no response and only 11 entries exhibiting greater than $50 \%$ response. For the mean across years, the mean response was $17 \%$, with 17 entries exhibiting no response and six entries exhibiting greater than $50 \%$ response. Transgressive segregation (individuals with greater response frequencies than H99) was observed in both years and in the mean across years, though most of these data points were dropped from the QTL analyses as outliers. The genotype and error variance components were 191 [95\% confidence interval (95\% $\mathrm{CI}=119-351)]$ and $352(95 \% \mathrm{CI}=279-459)$, respectively, for the non-transformed data and $2.6(95 \%$ $\mathrm{CI}=1.6-5.2)$ and $4.9(95 \% \mathrm{CI}=3.7-6.6)$, respectively, for the transformed data. The broad-sense heritabilities calculated on an entry mean-basis for Type I embryogenic callus response were $52 \%(90 \% \mathrm{CI}=12$ $52 \%)$ and $48 \%(90 \% \mathrm{CI}=10-51 \%)$ when calculated from the non-transformed and transformed data, respectively.

\section{QTL analysis}

QTL analysis was performed for the individual years and the mean across years for both the non-transformed and square-root transformed data. For the non-transformed data, outliers were observed for the individual years and the mean across years, with three, five, and two individuals removed from the 1997, 1998, and the mean across years analyses, respectively, while no outliers were observed in the transformed data.

For the non-transformed data, nine main effect QTL associated with $44 \%$ of the phenotypic variation were observed on chromosomes $1,2,3,5,6$, and 8 for the mean across years, while six and three QTL were observed for 1997 and 1998, respectively (Table 1; Fig. 3). All QTL observed in 1997 and 1998 were also observed in the mean across years, except one of the QTL on chromosome 5 in 1997 (umc27) and the QTL on chromosome 7 in 1998. Epistatic effects were significant but not consistent across the analyses. An additional epistatic interaction was significant in the mean across years, between loci on chromosomes 1 (isu73) and 6 (bnl5.47), but a model in which this interaction and the interaction between phi083 and phi061 were both significant could not be developed.

The main effect QTL observed in the mean across years for the non-transformed and transformed data did not differ (Table 2; Fig. 3). Only two main effect QTL each were observed for the transformed data in the 1997 and 1998 analyses, with only those from 1998 observed in the mean across trials. As with the nontransformed data, epistatic effects were significant but not consistent across the analyses of the transformed data, with two interactions observed in the mean across years that were not observed in the other years. For both interactions the presence of the $\mathrm{H} 99$ allele at both loci was associated with the highest percentage of Type I callus, while the presence of the Mo17 allele at one locus and the H99 allele at the other was associated with the lowest values.

The relative efficiencies of MAS alone, based on the adjusted $R^{2}$ from the mean across years models including epistatic interactions, were $\mathrm{RE}=0.99$ (0.92 when epistatic interactions are not included) 


\section{Chrom 1}

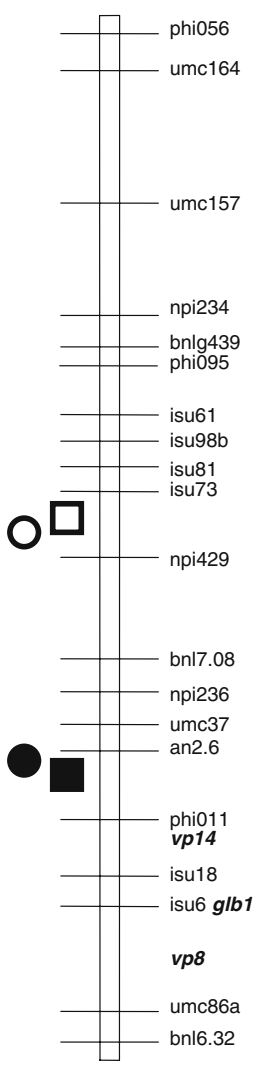

Chrom 6

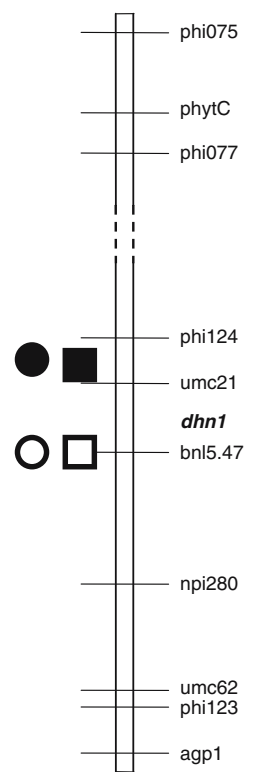

Chrom 2

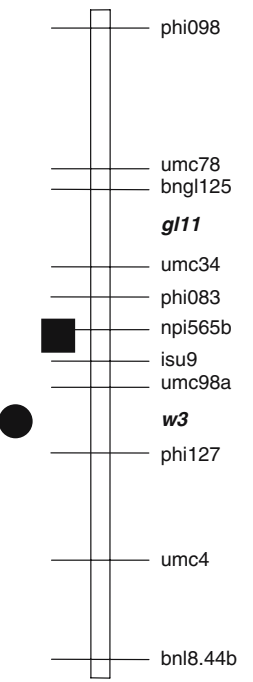

\section{$\square$ Cell totipotency}

OO Square root (Cell totipotency)

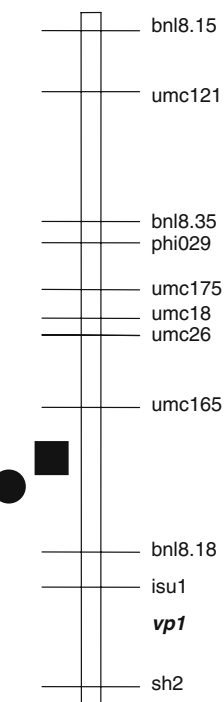

u

sh2

Chrom 4

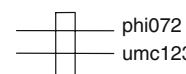

Chrom 7

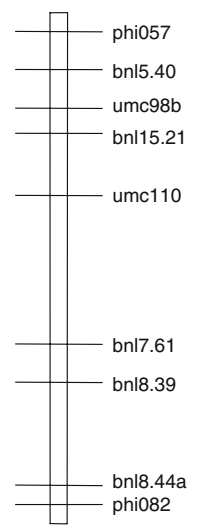

Chrom 8

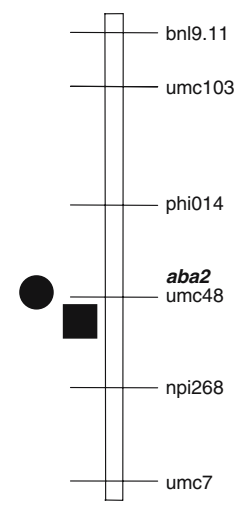

Chrom 9

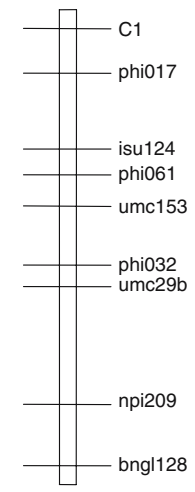

Chrom 5

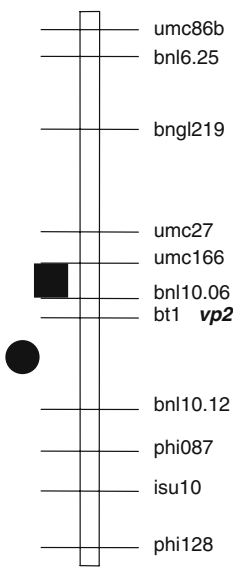

$10 \mathrm{cM}$

\section{Chrom 10}

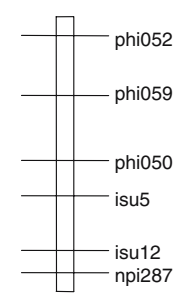

Fig. 3 Linkage map of Mo17 $\times$ H99 RILs population. Solid shapes (e.g., filled square) denote QTL for the mean across years for which the allele from $\mathrm{H} 99$ is associated with an increase in the trait, while outlined shapes (e.g. open square) denote QTL for the

for the non-transformed data and 0.98 ( 0.90 when epistatic interactions are not included) for the transformed data, while the relative efficiencies of MAS mean across years for which the allele from Mo17 is associated with an increase in the trait. Candidate genes are listed in bold italic: $\boldsymbol{v p}$, viviparous; $\boldsymbol{g l b 1}$, globulin; $\boldsymbol{g l 1 1}$, glossy; $\boldsymbol{w 3}$, white seedling; $\boldsymbol{d h n} \mathbf{1}$, dehydrin; $\boldsymbol{a b a 2}$, abscisic acid

combined with phenotypic data were $\mathrm{RE}=1.08$ for the non-transformed data and 1.09 for the transformed data. 
Table 1 Chromosomal locations, estimates of effects, and partial $R^{2}$ of QTL for induction of Type 1 callus

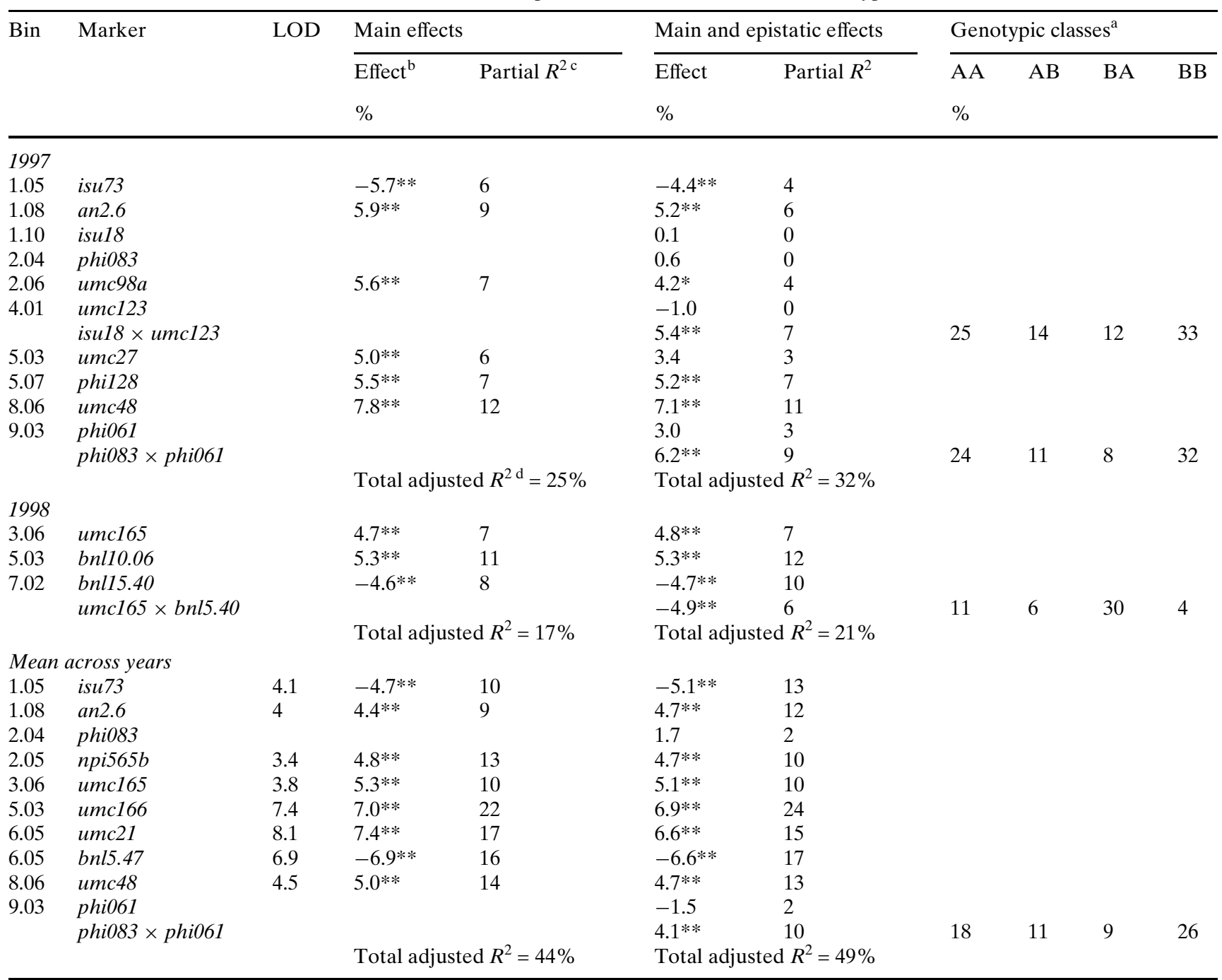

*, ** Significant at $P=0.10$ and 0.05 probability levels, respectively

${ }^{a}$ Means for individuals with Mo17 allele at both loci (AA), Mo17 allele at the first loci and H99 allele at second loci (AB), H99 allele at the first loci and Mo17 allele at second loci (BA), and H99 allele at both loci (BB)

${ }^{\mathrm{b}}$ The allele from H99 is associated with an increase $(+)$ or decrease $(-)$ in the value of the trait

${ }^{c}$ Percentage of phenotypic variation explained by the QTL, maintaining all other QTL effects fixed

${ }^{\mathrm{d}}$ Percentage of phenotypic variation explained by a model including all QTL as main effects and adjusted for the number of parameters in the model

\section{Discussion}

The square-root transformation resulted in more normalized data and removed the outliers but did not have much effect on the QTL analyses, with no differences observed between the main effect QTL observed in the non-transformed and transformed data for the mean across years. Epistatic interactions were observed for the mean across years for both the non-transformed and transformed data and contributed to significant increases in the adjusted $R^{2}$ for the models.

The presence of two QTL with opposite effects in the same bin on chromosome 6 may be the result of collinearity as opposed to the presence of two distinct QTL, since the distance between the QTL was only $20 \mathrm{cM}$ (Rawlings 1988). The additive effect of the QTL at bnl5.47 was negative when fit alone in the model but was only significant if the QTL at $u m c 21$ was present in the model and had a much greater impact on the partial $R^{2}$ of the QTL at $u m c 21$ than it did on the adjusted $R^{2}$ for the model. The epistatic interaction between isu 73 and bnl5.47 that was observed in the mean across years for the non-transformed data was only significant at $P=0.005$ in the mean across years analysis for the transformed data and was not included in the model. This interaction is unexpected in that the presence of 
Table 2 Chromosomal locations, estimates of effects, and partial $R^{2}$ of QTL for callus formation for the transformed data

\begin{tabular}{|c|c|c|c|c|c|c|c|c|c|c|}
\hline \multirow[t]{3}{*}{ Bin } & \multirow[t]{3}{*}{ Marker } & \multirow[t]{3}{*}{ LOD } & \multicolumn{2}{|c|}{ Main effects } & \multicolumn{2}{|c|}{ Main and epistatic effects } & \multicolumn{4}{|c|}{ Genotypic classes $^{\mathrm{a}}$} \\
\hline & & & Effect $^{\text {b }}$ & Partial $R^{2 \mathrm{c}}$ & Effect & Partial $R^{2}$ & AA & $\mathrm{AB}$ & BA & BB \\
\hline & & & \multicolumn{2}{|l|}{$\%$} & \multicolumn{2}{|l|}{$\%$} & \multicolumn{4}{|l|}{$\%$} \\
\hline \multicolumn{11}{|l|}{1997} \\
\hline 2.04 & phi083 & & & & -0.06 & 0 & & & & \\
\hline 2.06 & isu 9 & & & & -0.41 & 2 & & & & \\
\hline 5.07 & phi128 & 3.3 & $0.81 * *$ & 7 & $0.59 *$ & 5 & & & & \\
\hline \multirow[t]{2}{*}{7.03} & umc110 & & & & -0.27 & 1 & & & & \\
\hline & $i s u 9 \times u m c 110$ & & & & $0.88 * *$ & 10 & 4.1 & 1.7 & 2.8 & 4.8 \\
\hline \multirow[t]{2}{*}{9.02} & isu124 & 2.7 & $0.69 * *$ & 6 & $0.91 *$ & 4 & & & & \\
\hline & phi128 $\times$ isu124 & & & & $0.73 * *$ & 7 & 2.8 & 2.4 & 2.6 & 5.2 \\
\hline \multirow[t]{3}{*}{9.03} & phi061 & & & & -0.57 & 2 & & & & \\
\hline & phi083 $\times$ phi061 & & & & $0.90 * *$ & 11 & 3.8 & 2.2 & 2.5 & 5.1 \\
\hline & & & \multicolumn{2}{|c|}{ Total adjusted $R^{2 \mathrm{~d}}=8 \%$} & \multicolumn{2}{|c|}{ Total adjusted $R^{2}=30 \%$} & & & & \\
\hline \multicolumn{11}{|l|}{1998} \\
\hline 2.08 & umc4 & & & & 0.27 & 1 & & & & \\
\hline 3.06 & umc165 & 3.8 & $0.76^{* *}$ & 5 & $0.76^{* *}$ & 6 & & & & \\
\hline \multirow[t]{2}{*}{5.00} & $u m c 86 b$ & & & & 0.37 & 2 & & & & \\
\hline & $u m c 4 \times u m c 86 b$ & & & & $0.84 * *$ & 10 & 3.3 & 1.7 & 1.6 & 4.1 \\
\hline \multirow[t]{2}{*}{5.03} & bnl10.06 & 6.1 & $0.94 * *$ & 11 & $1.08 * *$ & 10 & & & & \\
\hline & & & \multicolumn{2}{|c|}{ Total adjusted $R^{2}=14 \%$} & \multicolumn{2}{|c|}{ Total adjusted $R^{2}=23 \%$} & & & & \\
\hline \multicolumn{11}{|c|}{ Overall } \\
\hline 1.06 & npi429 & 2.6 & $-0.60 * *$ & 7 & $-0.39 * *$ & 4 & & & & \\
\hline 1.08 & an 2.6 & 2.9 & $0.62 * *$ & 9 & $0.72 * *$ & 10 & & & & \\
\hline 1.10 & isu18 & & & & -0.16 & 1 & & & & \\
\hline 2.04 & phi083 & & & & 0.08 & 0 & & & & \\
\hline 2.06 & umc $98 a$ & 2.5 & $0.65 * *$ & 9 & $0.66 * *$ & 9 & & & & \\
\hline 3.06 & umc165 & 6.2 & $0.93 * *$ & 13 & $0.73^{* *} *$ & 10 & & & & \\
\hline \multirow[t]{2}{*}{4.01} & umc123 & & & & -0.17 & 1 & & & & \\
\hline & isu $18 \times$ umc123 & & & & $0.49 * *$ & 7 & 3.9 & 2.5 & 2.3 & 4.2 \\
\hline 5.04 & $b t 1$ & 2.7 & $0.98 * *$ & 22 & $0.92 * *$ & 23 & & & & \\
\hline 6.05 & umc21 & 6.2 & $1.02 * *$ & 15 & $0.76^{* * *}$ & 11 & & & & \\
\hline 6.05 & bnl5.47 & 3.6 & $-0.74 * *$ & 10 & $-0.64 * *$ & 9 & & & & \\
\hline 8.06 & umc48 & 2.5 & $0.54 * *$ & 6 & $0.65^{* *}$ & 9 & & & & \\
\hline \multirow[t]{3}{*}{9.03} & phi061 & & & & -0.22 & 2 & & & & \\
\hline & phi083 $\times$ phi061 & & & & $0.65^{* *}$ & 12 & 3.5 & 2.5 & 1.9 & 4.2 \\
\hline & & & \multicolumn{2}{|c|}{ Total adjusted $R^{2}=39 \%$} & \multicolumn{2}{|c|}{ Total adjusted $R^{2}=46 \%$} & & & & \\
\hline
\end{tabular}

*, ** Significant at $P=0.10$ and 0.05 probability levels, respectively

${ }^{a}$ Square-root transformed means for individuals with Mo17 allele at both loci (AA), Mo17 allele at the first loci and H99 allele at second loci (AB), H99 allele at the first loci and Mo17 allele at second loci (BA), and H99 allele at both loci (BB)

${ }^{b}$ Effects are based on square-root transformed values. The allele from H99 is associated with an increase (+) or decrease (-) in the value of the trait

${ }^{\mathrm{c}}$ Percentage of phenotypic variation explained by the QTL, maintaining all other QTL effects fixed

${ }^{\mathrm{d}}$ Percentage of phenotypic variation explained by a model including all QTL as main effects and adjusted for the number of parameters in the model

the Mo17 allele at both loci is associated with high trait values, when Mo17 is a poor responder, and is probably the result of a few outlying observations. Transgressive segregation was consistently observed in a few lines, but possible explanations include: (1) there are positive alleles for cell totipotency in Mo17, but they are swamped out by a much greater number of negative alleles, (2) there are negative alleles in H99 associated with inhibition of cell totipotency, while the alleles in Mo17 are neutral, or (3) there are more epistatic interactions between alleles from Mo17 and H99 than were observed herein. The study conducted herein cannot provide conclusive evidence for any one (or all) of these possibilities.

Previous reports on the genetics of embryogenic callus suggested that a few simply inherited genes were of major importance and that additive, non-additive, and maternal effects significantly affect the induction of the callus (Beckert and Qing 1984; Duncan et al. 1985; Hodges et al. 1985, 1986; Tomes 1985; Willman et al. 1989). While one major QTL $\left(R^{2}>20 \%\right)$ was observed on chromosome 5 (bin 5.03/4), most of the QTL had 
relatively small effects and epistatic effects were significant in both the individual years and the mean across years. Willman et al. (1989) reported significant epistatic variance for populations derived from crosses and backcrosses of A188 × Mo17 but not A188 × B73, and alleles from Mo17 had positive effects in some of the interactions observed herein.

Only one other report has been published on the locations of QTL associated with embryogenic callus, and that study evaluated induction of Type II callus (Armstrong et al. 1992). Conserved segments of A188 in a $\mathrm{BC}_{5}$ population of $(\mathrm{B} 73 \times \mathrm{A} 188) \times \mathrm{B} 73$ on chromosomes 1 (two segments), 2 , and 9 were evaluated for significance in an $\mathrm{A} 188 \times \mathrm{Mo17} \mathrm{F}_{2}$ population. Another segment was observed on chromosome 3, but was not tested in the $F_{2}$ population. Three segments (on chromosomes 1, 4, and 9) were significant for embryogenic callus in the $\mathrm{A} 188 \times \mathrm{Mo} 17 \mathrm{~F}_{2}$ population, none of which are linked to QTL observed herein. Interestingly the other two segments from the $\mathrm{BC}_{5}$ population, on chromosomes 2 and 3, appear to be linked (few common markers were used between the A188 $\times$ Mo17 $F_{2}$ population and the population herein) to QTL observed in the mean across years analyses herein. Differences in QTL observed between the two studies could be due to genetic heterogeneity between A188 and H99 and differences in the environments used for the studies.

Comparisons to microspore embryogenesis genetic studies, while confounded by differences in the traits, populations and environments, could provide some information about mechanisms of embryogenesis in tissue culture. Several studies have identified genetic regions associated with microspore embryogenesis, and of most interest are the studies that have used H99, which has very poor microspore embryogenesis response, as a parent in crosses to directly compare genomic regions associated with microspore and callus embryogenesis. Two studies used H99 in combination with Pa91 and FR16, inbreds with fair to good microspore embryogenesis response, and observed chromosomal regions associated with anther culture and ELS formation on chromosomes 1, 2, 3, 5, 6, 7, and 8 (Wan et al. 1992; Beaumont et al. 1995). The QTL observed herein for the mean across years in bins 1.08, 3.06, 5.03/ 4 , and 8.06 are in the same or bordering bins. Other studies used hybrid combinations that included lines that were selected for microspore culture response (e.g., DH5 and DH7) and localized regions associated with ELS formation on chromosomes 1, 3, 4, 5, 8, 9, and 10 (Cowen et al. 1992; Murgineux et al. 1994). The QTL observed herein for the mean across years in bins $1.06,1.08,3.06,5.03 / 4,8.06$, and 9.03 are in the same or bordering bins, and these comparisons across the various studies indicate possible common mechanisms for induction of embryogenic callus in immature embryos and ELS formation in cultured anthers.

Along with identifying genomic regions that could be useful in MAS, QTL studies can also be useful in identifying candidate genes that can provide a broader understanding of the genetics underlying the trait of interest. The viviparous mutants in maize are associated with decreased or deficient production of abscisic acid (ABA), a hormone involved in, among other roles, seed maturation and dormancy (Neill et al. 1986; Tan et al. 1997). The main and epistatic QTL observed herein in the mean across years for both non-transformed and transformed data in bins $1.08,1.10,2.04 / 5,3.06$, and $5.03 / 4$ are in the same or bordering bins as genes that have mutant alleles with viviparous phenotypes (e.g., vp1-vp14 and w3), while the epistatic QTL in bin 2.04 is in the same bin as glossy11, which sometimes has a viviparous phenotype (MaizeGDB, http://www.maizegdb.org/). In addition, the QTL on chromosome 8 is in the same bin as $a b a 2$, a candidate gene for ABA synthesis, and the QTL on chromosome 6 are in the same bin as dehydrin1, which is responsive to $\mathrm{ABA}$ and is expressed during dehydration. Beaumont et al. (1995) found that many QTL for induction of embryos during anther culture were also in the same chromosomal regions as genes that have viviparous mutant alleles, providing further evidence for the prominent role of ABA (or its absence) in the induction of embryos in vitro. Also, Marhic et al. (1998) observed that selection for anther culture response in maize resulted in a significant increase in the number of tillers per plant, which may have resulted from changes in ABA concentrations or sensitivities. It is not clear from these studies what exact role $\mathrm{ABA}$ has; the detection of so many QTL in close association with genes that have viviparous alleles does not necessarily mean that induction of embryos results from the suppression of ABA, and could be the opposite, since the alleles from highly responsive inbreds have not been specifically identified and characterized. Duncan et al. (2003) report that exposure of Type I callus to ABA or water stress induced the production of globulin 1 , a protein which is regulated by abscisic acid. The candidate gene for this protein $(g l b 1)$ is located in bin 1.09, in between the QTL observed herein in bins 1.08 and 1.10. Identification of allelic differences between Mo17 and H99 could provide further evidence of an association between genes related to ABA biosynthesis and induction of embryogenic callus, but several additional steps, such as detection of functional differ- 
ences between the alleles and transfer of the alleles into a recalcitrant inbred, would be necessary to verify that the genes were actually associated with induction of embryogenic callus.

Differences in isozyme patterns have been observed between embryogenic and non-embryogenic callus for glutamate dehydrogenase, acid phophatase, peroxidase, esterase, and malate dehydrogenase (Fransz et al. 1989; Rao et al. 1990). Candidate genes for glutamate dehydrogenase ( $g d h 1)$, acid phophatase (acp1), peroxidase (px13), and esterase (e3) are located in bins 1.11, $9.03,5.03$, and 3.05 , respectively, while two candidate genes for malate dehydrogenase, $m d h 4$ and $m d h 5$, are located in bins 1.08 and 5.03, respectively (MaizeGDB, http://www.maizegdb.org/). Whether these enzymes are directly associated with the induction of regenerable callus or simply the product of the physiological changes occurring as the callus becomes embryogenic is unclear, and the previous reports did not compare the isozyme patterns from embryogenic callus and zygotic embryos.

The efficiencies of MAS for the non-transformed and transformed data were about equal and were slightly lower than the efficiency of phenotypic selection alone, indicating that MAS would be useful only if it was less resource-intensive than phenotypic selection. The gain from using MAS and phenotypic selection combined was not enough to justify the resources involved.

QTL were observed on eight chromosomes herein, with both significant main and epistatic effects. Transformation of the data to produce a more normalized distribution did not have much effect on the QTL observed. While there was an obvious lack of consistency in the QTL observed when comparing 1997 to 1998 and either year to the mean across years, the fairly consistent localization of QTL in or near chromosomal bins associated with candidate genes, specifically candidate genes involved in ABA synthesis and sensitivity provides further evidence that the QTL are indeed real. Utilization of these QTL for MAS depends on the cost effectiveness of that approach as compared with phenotypic selection, but the QTL could prove useful in improving our understanding of the biology of induction of embryogenic callus, especially if allelic variability at the candidate genes is observed between the two inbred parents.

Acknowledgments This journal paper of the Iowa Agriculture and Home Economics Experiment Station, Ames, Iowa, Project No. 3134, was supported by Hatch Act and State of Iowa funds and The R. F. Baker Center for Plant Breeding.

\section{References}

Armstrong CL, Romero-Severson J, Hodges TK (1992) Improved tissue culture response of an elite maize inbred through backcross breeding, and identification of chromosomal regions important for regeneration by RFLP analysis. Theor Appl Genet 84:755-762

Austin DF, Lee M (1996) Comparative mapping in F2:3 and F6:7 generations of quantitative trait loci for grain yield and yield components in maize. Theor Appl Genet 92:817-826

Austin DF, Lee M (1998) Detection of quantitative trait loci for grain yield and yield components in maize across generations in stress and nonstress environments. Crop Sci 38:1296-1308

Austin DF, Lee M, Veldboom LR, Hallauer AR (2000) Genetic mapping in maize with hybrid progeny across testers and generations: grain yield and grain moisture. Crop Sci 40:3039

Beaumont VH, Rocheford TR, Widholm JM (1995) Mapping the anther culture response genes in maize (Zea mays L.). Genome 38:968-975

Beckert M, Qing CM (1984) Results of a diallele trial and a breeding experiment for in vitro aptitude in maize. Theor Appl Genet 68:247-251

Caravalho CHS, Bohorova N, Bordallo PN, Abreu LL, Valicente FH, Bressan W, Paiva E (1997) Type II callus production and plant regeneration in tropical maize genotypes. Plant Cell Rep 17:73-76

Cardinal A, Lee M, Moore KJ (2003) Genetic mapping and analysis of quantitative trait loci (QTL) affecting fiber and lignin content in maize. Theor Appl Genet 106:866-874

Cowen NM, Johnson CD, Armstrong K, Miller M, Woosley A, Pescitelli S, Skokut M, Belmar S, Petolino JF (1992) Mapping genes controlling in vitro androgenesis in maize using RFLP analysis. Theor Appl Genet 84:720-724

Duncan DR, Willams ME, Zehr BE, Widholm JM (1985) The production of callus capable of plant regeneration from immature embryos of numerous Zea mays genotypes. Planta 165:322-332

Duncan DR, Kriz AL, Paiva R, Widholm JM (2003) Globulin-1 gene expression in regenerable Zea mays (maize) callus. Plant Cell Rep 21:684-689

Fehr WR (ed) (1987) Principles of cultivar development, vol. I: Theory and technique. McGraw-Hill, NY

Fransz PF, de Ruijter NCA, Schel JHN (1989) Isozymes as biochemical and cytochemical markers in embryogenic callus cultures of maize (Zea mays L.). Plant Cell Rep 8:67-70

Green CE, Phillips RL (1975) Plant regeneration from tissue cultures of maize. Crop Sci 15:417-421

Green CE, Rhodes CA (1982) Plant regeneration in tissue cultures of maize. In: Sheridan WF (eds) Maize for biological research. Plant Molecular Biology Association, Charlottesville, pp. 367-372

Hodges TK, Kamo KK, Becwar MR, Schroll S (1985) Regeneration of maize. In: Zaitlin M, Day P, Hollaender A, Wilson C (eds) Biotechnology in plant science: relevance to agriculture in the eighties. Academic, Orlando, pp. 15-33

Hodges TK, Kamo KK, Imbrie CW, Becwar MR (1986) Genotype specificity of somatic embryogenesis and regeneration in maize. Biotechnology 4:219-223

Holland JB (1998) EPISTACY: A SAS program for detecting two-locus epistatic interactions using genetic marker information. J Hered 89:374-375

Holland JB, Moser HS, O'Donoughue LS, Lee M (1997) QTLs and epistasis associated with vernalization responses in oat. Crop Sci 38:1306-1316 
Knapp SJ, Stroup WW, Ross WM (1985) Exact confidence intervals for heritability on a progeny mean basis. Crop Sci 25:192-194

Krakowsky MD, Lee M, Woodman-Clikeman WL, Long MJ, Sharpova N (2004) QTL mapping of resistance to stalk tunneling by the European corn borer in RILs of maize population B73 $\times$ De811. Crop Sci 44:274-282

Lande R, Thompson R (1990) Efficiency of marker-assisted selection in the improvement of quantitative traits. Genetics 124:743-756

Lu C, Vasil K, Ozias-Akins P (1982) Somatic embryogenesis in Zea mays L. Theor Appl Genet 62:109-112

Marhic A, Antoine-Michaud S, Bordes J, Pollacsek M, Murigneux A, Beckert M (1998) Genetic improvement of anther culture response in maize: relationships with molecular, Mendelian, and agronomic traits. Theor Appl Genet 97:520525

Murigneux A, Bentolila S, Hardy T, Baud S, Guitton C, Jullien H, Ben Tahar S, Freyssinet G, Beckert M (1994) Genotypic variation of quantitative trait loci controlling in vitro androgenesis in maize. Genome 37:970-976

Neill SJ, Horgan R, Parry AD (1986) The carotenoid and abscisic acid content of viviparous kernels and seedlings of Zea mays L. Planta 169:87-96

Papst C, Bohn M, Utz HF, Melchinger AE, Klein D, Eder J (2004) QTL mapping for European corn borer resistance (Ostrinia nubilalis $\mathrm{Hb}$.), agronomic and forage quality traits of testcross progenies in early-maturing European maize (Zea mays L.) germplasm. Theor Appl Genet 108:1545-1554

Phillips RL, Somers DA, Hibberd KA (1988) Cell/tissue culture and in vitro manipulation. In: Sprague CF, Dudley JW (eds) Corn and corn improvement, 3rd edn. American Society of Agronomy Inc., Madison, WI pp. 245-288
Rao KV, Suprasanna P, Reddy GM (1990) Biochemical changes in embryogenic and non-embryogenic calli of Zea mays L. Plant Sci 66:127-130

Rawlings JO (1988) Applied regression analysis: a research tool. Wadsworth and Brooks, Pacific Grove, CA pp. 162-165

SAS Institute Inc (1999) SAS OnlineDoc ${ }^{\circledR}$, Version 8. SAS Institute, Inc, Cary, NC

Tan BC, Schwartz SH, Zeevaart JAD, McCarty DR (1997) Genetic control of abscisic acid biosynthesis in maize. Proc Natl Acad Sci U.S.A. 94:12235-12240

Tomes DT (1985) Opportunities and limitations of the genotypic influences on establishment and plant regeneration from callus an cell cultures of crop species. In: Zaitlin M, Day P, Hollaender A, Wilson C (eds) Biotechnology in plant science: relevance to agriculture in the eighties. Academic, Orlando, FL pp. 3-14

Tomes DT, Smith OS (1985) The effect of parental genotype on initiation of embryogenic callus from elite maize (Zea mays L.) germplasm. Theor Appl Genet 70:505-509

Utz HF, Melchinger AE (1996) PLABQTL: a program for composite interval mapping of QTL. J Quant Trait Loci 2:1

Veldboom LR, Lee M, Woodman WL (1994) Molecular markerfacilitated studies in an elite maize population: I. Linkage analysis and determination of QTL for morphological traits. Theor Appl Genet 88:7-16

Wan Y, Rocheford TR, Widholm JM (1992) RFLP analysis to identify putative chromosomal regions involved in the anther culture response and callus formation in maize. Theor Appl Genet 85:360-365

Willman MR, Schroll SM, Hodges TK (1989) Inheritance of somatic embryogenesis and plantlet regeneration from primary (Type I) callus in maize. In Vitro Cell Dev Biol 25:95-100 